\title{
Synaptophysin and Hematoxylin Staining Method
}

National Cancer Institute

\section{Source}

National Cancer Institute. Synaptophysin and Hematoxylin Staining Method. NCI

Thesaurus. Code C28415.

Samples counterstained with hematoxylin following Synaptophysin staining. 